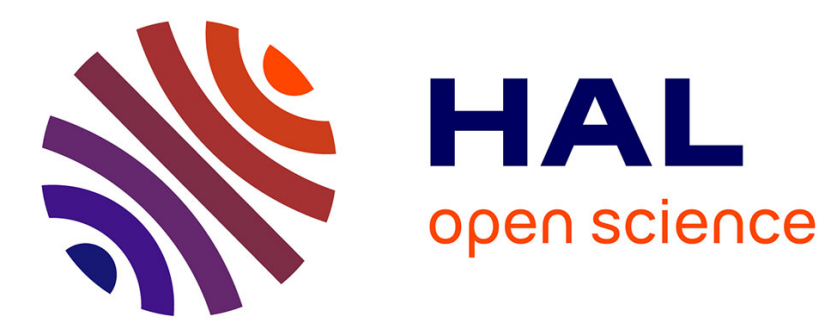

\title{
Viscosity measurements of thin polymer films from reflow of spatially modulated nanoimprinted
}

Etienne Rognin, Stéfan Landis, Laurent Davoust

\section{To cite this version:}

Etienne Rognin, Stéfan Landis, Laurent Davoust. Viscosity measurements of thin polymer films from reflow of spatially modulated nanoimprinted. Physical Review E: Statistical, Nonlinear, and Soft Matter Physics, 2011, 84 (4), 10.1103/PhysRevE.84.041805 . hal-00664542

\section{HAL Id: hal-00664542 \\ https://hal.science/hal-00664542}

Submitted on 19 Apr 2020

HAL is a multi-disciplinary open access archive for the deposit and dissemination of scientific research documents, whether they are published or not. The documents may come from teaching and research institutions in France or abroad, or from public or private research centers.
L'archive ouverte pluridisciplinaire HAL, est destinée au dépôt et à la diffusion de documents scientifiques de niveau recherche, publiés ou non, émanant des établissements d'enseignement et de recherche français ou étrangers, des laboratoires publics ou privés. 


\title{
Viscosity measurements of thin polymer films from reflow of spatially modulated nanoimprinted patterns
}

\author{
Etienne Rognin* and Stefan Landis \\ CEA-LETI-Minatec Campus, 17 rue des martyrs, F-38054 Grenoble Cedex 9, France \\ Laurent Davoust \\ SIMAP/EPM, Grenoble Institute of Technology, 1130 rue de la piscine, F-38400 Saint Martin d'Hères, France
}

(Received 10 June 2011; revised manuscript received 20 September 2011; published 14 October 2011)

\begin{abstract}
We present a method to measure the viscosity of polymer thin films. The material is spin coated onto a silicon substrate and specially designed nanopatterns are imprinted on the film using thermal nanoimprint. A brief reflow is performed during which patterns flow under surface tension. Spectral densities of the topology before and after annealing are compared and the rheologic properties, such as viscosity, are extracted as fitting parameters of an evolution model. Contrary to previous similar approaches, emphasis was put on the spatial description rather than the temporal decay of the patterns. We used this method to measure the viscosity of polystyrene for two molecular weights at various temperatures and successfully recovered results of previous authors.
\end{abstract}

\section{INTRODUCTION}

Nanoimprint lithography (NIL) emerged in the mid-1990s [1] and has been rapidly considered as a high-resolution and high-throughput patterning technique. Despite a huge development of imprinting equipment, stamp manufacturing processes, imprint processes, dedicated materials, and metrology approaches, a complete simulation toolbox of NIL is still lacking. To propose a complete modeling approach several issues are still pending: The complex mechanical stamp deformations during imprinting $[2,3]$ are not yet fully understood; the flow properties of melted resists within nanometric cavities are not well known and resist property characterizations at such small scales are not always possible; the stamp-resist adhesion and friction mechanisms either in parallel or peeling [4] demolding schemes also need to be introduced to overcome the resist fracture defect [5]. In this paper we propose to address the issue related to the resist characterization at the submicron scale in order to provide the resist properties data sheet needed to model the imprint process. Characterization of flow properties of melted polymers at the macroscopic scale is now a quite easy task to perform from classical measurement devices devoted to volume rheology. In NIL processes, resist film thickness ranges from several tens of nanometers up to several hundreds of nanometers. In such configurations, surface phenomena increasingly play a decisive role with significant deviation for viscous flows properties [6].

To measure the properties of fluids at the nanoscopic scale, numerous methods have been presented. An early approach is based on the nucleation and growth rate of holes in a dewetting polymer film [7-10]. Although this approach yields consistent results, it also raises both theoretical and experimental issues. The dynamics of a moving contact line is indeed largely simplified [11]. Moreover, a limited range of film thickness and substrate material can be used in order to observe the expected dewetting dynamics. A second approach is based on measuring the resistance of the flow around an immersed

*etienne.rognin@cea.fr
AFM tip [12-16]. This method only measures mechanical properties at high frequency and depends to a large extent on an accurate knowledge of the tip shape. Finally, a third approach, closely related to what is presented here, is to measure the relaxation dynamics of a surface relief. We can distinguish between methods where the topography is induced by thermal fluctuations [9,17-19] and methods where the topography is imprinted by a mold, using common nanoimprint techniques $[20,21]$. The dynamics is probed either by using x-ray photon correlation microscopy or more generally by light scattering techniques. In this approach, attention is given to the time evolution of the fundamental spatial frequency of the shape, and viscosity is determined from the measured relaxation time. In many cases, neither the exact topology nor the thermal dependance of the optical index of the material is known, and delicate data processing is needed.

In this paper, we present a method to measure the viscosity of a polymer thin film from the reflow of nanoimprinted patterns. We focus our efforts on the accurate spatial determination of the surface of the film, rather than on its temporal evolution, and we extend the method formulated by Leveder et al. [22,23]. Atomic force microscopy (AFM) measurements with a spatial resolution lower than $1 \mathrm{~nm}$ are used to extract the spectral density of the topography at two different times of the reflow. The mechanical properties of the film are extracted from the comparison of these two densities, by means of a robust analytical model. The imprinted patterns are specially designed to provide relevant spectral densities, in order to perform measurement of a large viscosity range and at different temperatures.

\section{METHOD}

\section{A. Overview}

In this section, we present the main steps of the method, drawn in Fig. 1. The material (polymer) is spin coated onto a silicon substrate, panel (a), and the thickness of the film is measured by ellipsometry. We begin with imprinting the film by thermal nanoimprint [1,3], panel (b), using a 
(a) Material coating

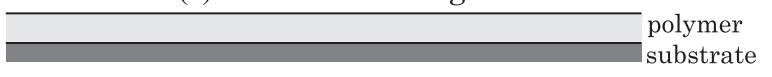

(b) Nanoimprint

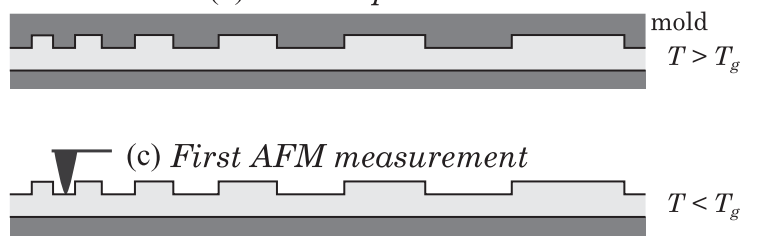

(d) Reflow

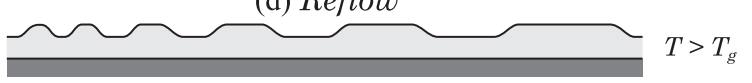

(e) Second AFM measurement

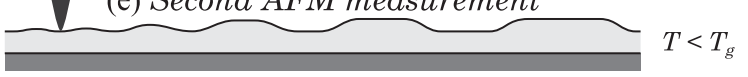

FIG. 1. Main steps of the viscosity measurement method.

specially designed mold which will be described hereafter. After demolding at room temperature, a first measurement of the imprinted profile is done by AFM, panel (c). The film is then heated at a definite temperature above the glass transition temperature $\left(T_{g}\right)$ during a definite time, panel (d). The film is then rapidly cooled down and the reflowed profile is again measured by AFM, panel (e). Spectral densities of the profiles are computed using standard Fourier transform algorithms, and the sought viscosity is a fitting parameter of an evolution model for the spectral density of the topology, described in the next section.

At temperatures above $T_{g}$, the film becomes viscous and begins to flow. At submicron scale and for highly viscous fluids, both gravity and inertia are negligible: The flow is only driven by surface tension (and in some cases van der Waals forces) [24]. The film reaches a steady state when the surface energy of the free interface is minimum. In other words, if no dewetting occurs, then the film becomes completely flat. During the reflow process, the shape of the interface evolves with the balance between the surface tension, which tends to smooth the bumps and protrusions, and the viscous shear, which damps the flow. Locally, the more curved the interface is, the higher the pressure inside the fluid is: Short-length features of high curvature are the first elements to disappear. Moreover, long-length general shapes flatten out more slowly because the fluid must flow over longer distances under high shear. These local behaviors produce a large-scale consequence of the reflow which is a low-pass filtering of the topology of the film. We precisely take advantage of this spatial filtering to extract the viscosity of the material.

\section{B. Model}

We model a fluid film which is supported by a flat solid substrate, typically made of silicon (Fig. 2). The thickness of the film is described by the local and instantaneous function $h(x, y, t)$, which we write as a sum of two parts: $h=h_{0}+$ $\tilde{h}(x, y, t)$, where $h_{0}$ is the mean thickness of the film (over time and space). In order to derive a simple analytical model for the flow, we assume that the deviation of the free surface

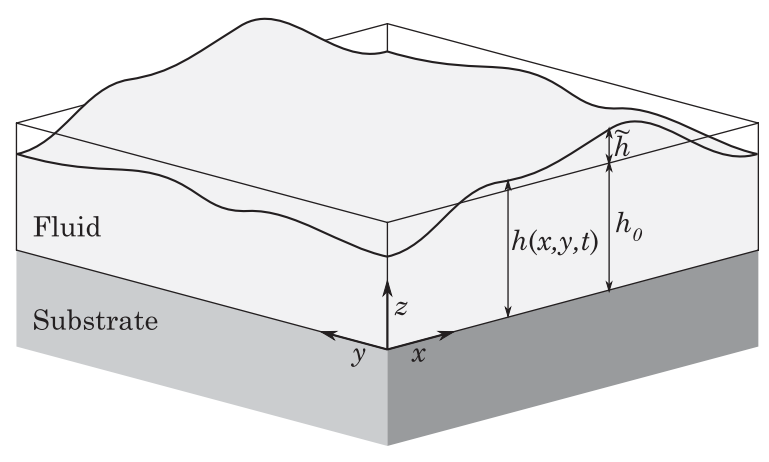

FIG. 2. Schematic of a thin fluid film.

from the mean thickness value is small, in other words that $\tilde{h} / h_{0} \ll 1$. Note that the geometric condition $\tilde{h} / h_{0} \ll 1$ is only a condition on the amplitude of the deformation, not on the pattern wavelength (or wave vector), as it usually would be in the lubrication approximation $[25,26]$.

A flow model under this kind of setup was previously solved by Henle and Levine [27] for the characterization of spontaneous capillary waves. Here we summarize their approach and in addition we take the van der Waals forces into account. We make the fundamental hypothesis that we can describe our microfluidic systems by the laws of continuum mechanics. Assuming that the fluid is incompressible, the mass conservation is formulated by

$$
\nabla \cdot \mathbf{v}=0
$$

where $\mathbf{v}$ is the fluid velocity. At scales smaller than the capillary length, we can neglect body force terms (such as gravity) compared to surface forces. Moreover, since the Reynolds number of the flow is extremely low $\left(\operatorname{Re} \sim 10^{-12}\right)$, the viscous terms in the equation of motion overcome inertial terms. The dynamics of the flow is thus described by the Stokes equation:

$$
\nabla p=\nabla \cdot \mathbb{T},
$$

where $p$ is the pressure field and $\mathbb{T}$ is the viscous stress tensor. The hydrodynamics problem is closed by a behavior law for the material. Polymer melts are known to have a nonlinear behavior at high strain, such as shear thinning. However, in the limit of small strain, the response of the material at a given frequency $\omega$ can be described by the complex shear modulus $G(\omega)$ [28]. In the case of a linear behavior, the Stokes equation in the frequency domain becomes

$$
\nabla p(\omega)=i \frac{G(\omega)}{\omega} \nabla^{2} \mathbf{v}(\omega)
$$

We describe below a method to solve the flow from a linear perturbative method. We begin with transforming the flat coordinates $(x, y)$ into the Fourier domain $\mathbf{k}=\left(k_{x}, k_{y}\right)$, and the time into the frequency domain. The height coordinate $z$ is left in the real space. Each variable depends then on $k_{x}$, $k_{y}, z$, and $\omega$, but for simplicity most of these dependencies are not written. The horizontal part of the velocity is written $\mathbf{u}=\left(v_{x}, v_{y}\right)$. We also write the $z$ derivative $\partial v / \partial z=v^{\prime}$. Under this transformation, we have for the continuity equation

$$
\mathbf{k} \cdot \hat{\mathbf{u}}=i \hat{v}_{z}^{\prime}
$$


and for the equation of motion

$$
\begin{aligned}
& \frac{\omega \hat{p}}{G} \mathbf{k}=-k^{2} \hat{\mathbf{u}}+\hat{\mathbf{u}}^{\prime \prime}, \\
& \frac{\omega \hat{p}^{\prime}}{i G}=-k^{2} \hat{v}_{z}+\hat{v}_{z}^{\prime \prime},
\end{aligned}
$$

where $k=\sqrt{\mathbf{k}^{2}}$.

We now focus on boundary conditions. At the bottom of the fluid, the velocity must vanish (no-slip condition):

$$
\hat{\mathbf{v}}(z=0)=\mathbf{0} .
$$

By assuming that the film is almost flat $\left(\tilde{h} / h_{0} \ll 1\right)$, the boundary condition at the fluid-air interface can be linearized. The pressure at that interface is given by the sum of two contributions: the surface tension and the van der Waals forces (disjoining pressure) [25,26]. The linearized condition is

$$
\hat{p}\left(z=h_{0}\right)=\left(\gamma k^{2}+\frac{A}{2 \pi h_{0}^{4}}\right) \hat{h}(\mathbf{k}, \omega),
$$

where $\gamma$ is the surface tension and $A$ is the Hamaker constant. Moreover, as no shear stress is supposed to apply along the interface, we can write

$$
\frac{i G(\omega)}{\omega}\left(i \hat{v}_{z} \mathbf{k}+\hat{\mathbf{u}}^{\prime}\right)_{z=h_{0}}=\mathbf{0} .
$$

In addition, as a linearized kinematic condition at the fluid-air interface, the vertical velocity equals the growth rate of the thickness:

$$
-i \omega \hat{h}(\mathbf{k}, \omega)=\hat{v}_{z}\left(z=h_{0}\right) .
$$

Finally, we say that there is no stress applied on the material at time before the reflow $(t<0)$; in other words there is no residual elastic stress caused by the imprint (see Sec. III B).

All the elements are set to solve the equation of motion. This calculation is carried out in the Appendix. We finally get the dispersion relation between the wave vector $k$ and the complex frequency $\omega$ :

$$
\frac{h_{0} G(\omega)}{\gamma}+f\left(k h_{0}, \mathrm{Ha}\right)=0,
$$

where $f$ is a dimensionless function of the normalized wave vector $k h_{0}$ and of the dimensionless Hamaker number $\mathrm{Ha}=$ $A / 2 \pi \gamma h_{0}^{2}$. The function $f$ is given by

$$
f\left(k h_{0}, \mathrm{Ha}\right)=\frac{\sinh k h_{0} \cosh k h_{0}-k h_{0}}{2 k h_{0} \cosh ^{2} k h_{0}}\left(k^{2} h_{0}^{2}+\mathrm{Ha}\right) .
$$

Note that Ha may be either positive or negative, depending on the sign of $A$ [29]. When $A$ is negative, the film is known to be unstable and Ha quantifies the competition between the surface tension which smooths the film and the van der Waals forces which dewet it. Features whose wave vectors are smaller than $k_{c}=\sqrt{|\mathrm{Ha}|} / h_{0}$ tend to grow exponentially leading to the rupture of the film. In our experimental setup, Ha $\sim 10^{-5}$, we have $k_{\min } / k_{c} \sim 10^{3}$, so $k_{\min } \gg k_{c}$, which is why van der Waals forces do not cause the rupture of the film, and $\mathrm{Ha}$ is rather considered as a small (often negligible) corrective parameter.

In the case of a simple Newtonian fluid, $G(\omega)=-i \omega \eta$ where $\eta$ is the viscosity. Then Eq. (7) has only one root on the imaginary axis, which means that all the modes are purely damped (i.e., they do not propagate). The decay time $\tau(k)$ for each mode of wave vector $k$ is extracted from the dispersion relation and leads to

$$
i \omega=\frac{1}{\tau(k)}=\frac{\gamma f\left(k h_{0}, \mathrm{Ha}\right)}{\eta h_{0}} .
$$

From this result, we can write the evolution of the thickness:

$$
\hat{h}(\mathbf{k}, t)=\hat{h}(\mathbf{k}, 0) \exp \left(-\frac{t}{\tau(k)}\right) .
$$

Equation (8) shows that the reflow of the film is equivalent to a low-pass Fourier spatial filter. The filter kernel exp $[-t / \tau(k)]$ is given by the physical parameters of the problem. In particular, when the van der Waals forces are not significant, the decay time $\tau(k)$ has two known asymptotic regimes. When $k h_{0} \ll 1$, referred to as the lubrication regime, we have $\tau_{k} \sim 3 \eta / \gamma h_{0}^{3} k^{4}$. This scaling of the decay time regarding both $k$ and $h_{0}$ was experimentally verified by Leveder et al. [23]. On the other hand, when $k h_{0} \gg 1$, we find $\tau_{k} \sim 2 \eta / \gamma k$ which is the dispersion relation for bulk flows reported by Hamdorf [20]. Note that in the case with $h_{0} \rightarrow \infty$, the decay time does not depend on the thickness of the film. It is therefore important to take both regimes into account with the full dispersion relation Eq. (7) since in the data analysis a large range of normalized wave vectors $k h_{0}$ is involved.

\section{Mold design}

From Eq. (8) we can see that there are two ways to measure the kernel function and then extract the viscosity. The first one, used by $[9,17-21,23]$, is to follow the amplitude $h\left(k_{1}, t\right)$ of the surface for a given mode $k_{1}$, as a function of time $t$. This is done with periodical patterns, focusing on the decrease of the fundamental frequency. An exponential decrease is found, and the model is fitted to that data. This is straightforward if there is a real-time analysis, such as scatterometry. However, along with the issues raised in the introduction section of this paper, an additional problem is that, due to the steep variation of the decay time with respect to the wave vector and the mean thickness, one can only measure viscosity with a range of a single decade, for a given pattern and thickness, and assuming reasonable experimental times. Multiple patterns are therefore needed to cover a wide range of viscosity. Another issue is the fact that one measurement flattens the topology, hence several samples have to be used in order to perform measurements at different temperatures.

The other way to obtain the kernel function is to measure the decay of all the modes for a given time $t_{a}$ of annealing, in other words, to measure $h(k, 0)$ and $h\left(k, t_{a}\right)$ as a function of wave vector $k$. In order to realize this effectively, we need a topology with a rich spectral density but short enough to be measured by a single AFM scan. We present below a patented approach to designing a mold with such a profile.

We can define a pattern shape $m(x)$ of length $L$ and of depth $2 \tilde{m}$ by the modulation function

$$
m(x)=\tilde{m} \operatorname{sgn}\left[\cos \left(\int_{0}^{x} k(\xi) d \xi\right)\right]
$$

where $k(\xi)$ is the local wave vector. Note that if $k(\xi)=k_{1}$ (the pattern is periodical), then $m(x)=\tilde{m} \operatorname{sgn}\left[\cos k_{1} x\right]$. The 
choice of the function $k(\xi)$ is essential to determine the spectral density. Here we give two examples of local wave vector functions. The first one aims at providing a uniform spectral density, so that the same weight is given to low and high frequencies. Its expression is given by

$$
k(x)=k_{\max }+\left(k_{\min }-k_{\max }\right) \frac{x}{L},
$$

where $k_{\min }$ and $k_{\max }$ are, respectively, the minimum and the maximum local wave vectors. This type of profile is particularly relevant if the measurements are performed by AFM. However, one could also use other instruments, such as optical or confocal microscopy. A second expression is thus needed to enhance visual contrast between reflowed areas (high frequencies) and slower dynamics areas (low frequencies). Such profile can be defined by

$$
k(x)=k_{\max }\left(\frac{k_{\min }}{k_{\max }}\right)^{x / L} .
$$

In the next section we present some experimental application and results of the method.

\section{EXPERIMENT}

Experiments were carried out on polystyrene samples. The following subsections cover many aspects of the experimental process. The first one is dedicated to material properties and preparation. The second and third subsections go through the imprint and reflow of the samples. The last one is a comment on the AFM measurements. Results are finally presented in the next section.

\section{A. Materials}

Low molecular weight $\left(M_{w}=30 \mathrm{~kg} \cdot \mathrm{mol}^{-1}\right.$ and $M_{w}=$ $\left.130 \mathrm{~kg} \cdot \mathrm{mol}^{-1}\right)$ monodisperse $\left(M_{w} / M_{n}<1.06\right)$ polystyrene (PS 30k and PS 130k) solution is spin coated onto 8-inch silicon substrates. Prior to coating PS, substrates were rinsed with a hydroxylamine/aminethoxyethanol (60\%/40\%) bath during $600 \mathrm{~s}$ at $343 \mathrm{~K}$, and a $\mathrm{O}_{2} / \mathrm{N}_{2} \mathrm{H}_{2}(2150 \mathrm{sccm} / 240 \mathrm{sccm})$ plasma during $180 \mathrm{~s}$ at $543 \mathrm{~K}$ was performed in order to obtain a homogeneous 4-nm-thick SiOx layer. The glass transition temperature $\left(T_{g}=102 \pm 1{ }^{\circ} \mathrm{C}\right)$ is measured by differential scanning calorimetry (DSC). Thicknesses of coated polystyrene films were $150 \pm 1 \mathrm{~nm}$ and measured by ellipsometry.

The surface tension of polystyrene melts varies with both molecular weight and temperature. However, this variation is small - in contrast with the variation of viscosity - for entangled polystyrene and for the range of temperature investigated in this paper $\left(120^{\circ} \mathrm{C}\right.$ to $\left.180^{\circ} \mathrm{C}\right)$. Typical values (extrapolated for our molecular weights from tabulated values [28]) are $\gamma @ 120^{\circ} \mathrm{C}=33.3 \mathrm{mN} \cdot \mathrm{m}^{-1}$ and $\gamma_{@ 180^{\circ} \mathrm{C}}=29.0 \mathrm{mN} \cdot \mathrm{m}^{-1}$ for PS $30 \mathrm{k}$, and $\gamma_{@ 120^{\circ} \mathrm{C}}=34.8 \mathrm{mN} \cdot \mathrm{m}^{-1}$ and $\gamma_{1} 180^{\circ} \mathrm{C}=$ $30.4 \mathrm{mN} \cdot \mathrm{m}^{-1}$ for PS $130 \mathrm{k}$. As we do not know the exact thermal dependency, it is reasonable to take a constant value $\gamma=32 \pm 3 \mathrm{mN} \cdot \mathrm{m}^{-1}$ for both molecular weights.

\section{B. Imprint}

The imprints of the patterns were made at 13 bars of pressure and at temperature $T_{g}+80^{\circ} \mathrm{C}$ where the elastic stress relaxes quickly (measured by disk rheometry to be less than $1 \mathrm{~s}$ ) in comparison to the imprint time (which was at least $30 \mathrm{~min}$ ). The uniformity of the residual layer is ensured because, first, the depth of the pattern is small compared with the initial thickness of the film, second, the pattern is locally isodense (no large scale flow of mass), and third, the total length of the pattern $(40 \mu \mathrm{m})$ is small compared to the thickness of the mold $(750 \mu \mathrm{m})$ so that no bending of the mold occurred [3]. Demolding was performed at room temperature.

\section{Heating and quench}

The model we previously described requires that the polymer film changes instantaneously from a glassy solid state below $T_{g}$ to a fluid state at a fixed temperature above $T_{g}$. From an experimental point of view, this cannot be strictly achieved because of thermal inertia of the sample. However, we can choose the total reflow duration so that the time in a non-steady-state can be neglected, at the cost of an appropriate error estimate on the final viscosity measurement.

In our experiments, the heating of the sample was done with a hot plate with $1{ }^{\circ} \mathrm{C}$ precision. The energy is transferred to the sample through the contact surface. The quench, i.e., the rapid cooling, was performed by using a cooling plate thermalized at room temperature. Given that the sample is thin (no significant heat flux at the edges) and that the flux through both sides is homogeneous, this quench is a one-dimensional thermal transfer through the thickness of the sample. One way to address the heat transfer dynamics is to compute the order of magnitude for the time scale of each transfer phenomenon. Given by an energy balance, such a time scale takes the general form $\tau=e C / H$, where $e$ is the thickness of the sample, $C$ is the heat capacity of the sample per unit of volume, and $H$ is the thermal conductance per unit of surface related to the (linearized) transfer phenomenon. In our case, there are three types of transfers for which we can give typical time scales: the conduction of heat inside the wafer $\left(\tau_{\mathrm{cd}} \sim 1 \mathrm{~ms}\right)$, the free convection and radiation on the top side $\left(\tau_{\mathrm{cv}-\mathrm{rd}} \sim 120 \mathrm{~s}\right)$, and the contact transfer through the bottom side $\left(\tau_{\mathrm{tc}} \sim 1 \mathrm{~s}\right)$.

The fact that $\tau_{\mathrm{cd}} \ll \tau_{\text {tc }}$ demonstrates that the temperature is always homogeneous inside the sample. In addition, $\tau_{\text {tc }} \ll$ $\tau_{\mathrm{cv}-\mathrm{rd}}$ means that free convection and radiation can be neglected in the heat transfer dynamics. Finally, the characteristic time governing the temperature of the sample is $\tau_{\mathrm{tc}} \sim 1 \mathrm{~s}$; therefore the sample reaches a steady state within a few seconds at most. According to the foregoing scaling analysis, if we choose a reflow duration of at least $5 \mathrm{~min}$, it is reasonable to consider that the transition is instantaneous.

\section{AFM measurements}

For the AFM measurements we used Nanosensors AR5NCHR tips in tapping mode. These tips have high aspect ratios (7:1) and low radii $(<15 \mathrm{~nm})$. The typical resonance frequency was $330 \mathrm{kHz}$.

All AFM measurements are done at room temperature; thus there is essentially no issue of temperature drift. It is however well known that tip geometry induces a distortion in the measured image [30,31]. This effect is a nonlinear convolution which can have a direct influence on the spectral 
density, and finally on the viscosity measurement. The mathematical treatment of a nonlinear deconvolution is not straightforward and requires an accurate knowledge of the tip shape. Without special equipment dedicated to this issue it is thus of importance to minimize such convolution. In our case, this is ensured by the low aspect ratio of the imprinted profiles, which means that the tip can fully enter the trenches and that the shadows induced by the steep steps are negligible. It is also possible to perform a brief reflow just after demolding in order to soften the edges of the profile, and thus reduce or suppress the tip convolution. This new state may be therefore used as the initial one.

\section{RESULTS AND DISCUSSION}

Figure 3 gives an example of a viscosity measurement done with the method described above. A 150-nm-thick PS 30k was annealed at $120^{\circ} \mathrm{C}$ during $5 \mathrm{~min}$. The AFM measurements reported in Fig. 3(a) emphasize the low-pass filtering behavior of the reflow, since the high-frequency part of the profile completely vanished after annealing. The ratio of Fourier transforms of both profiles plotted in Fig. 3(b) gives the kernel function of the filter whose expression is given by Eq. (8). The error bars are drawn assuming a $1 \mathrm{~nm}$ rms Gaussian noise for each AFM measurement. The large uncertainty area for the high-order modes accounts for the fact that no information can be extracted from features which have completely flowed. From the data points it is then possible to compute the number $\mathrm{Ca}=\eta h_{0} / t \gamma$ as a fitting parameter of the kernel function $\exp \left[-f\left(k h_{0}\right) / \mathrm{Ca}\right]$. The viscosity is readily obtained with the knowledge of the other physical parameters.

Let us now make several remarks about the accuracy of the method. The number $\mathrm{Ca}$ is given with a relative error $\sigma_{\mathrm{Ca}}=2 \%$ which comes from the residuals of the fitting. The uncertainty of the other parameters is, in our experiments (see previous section), $\sigma_{h_{0}}=0.7 \%$ for the mean thickness, $\sigma_{\gamma}=9 \%$ for the

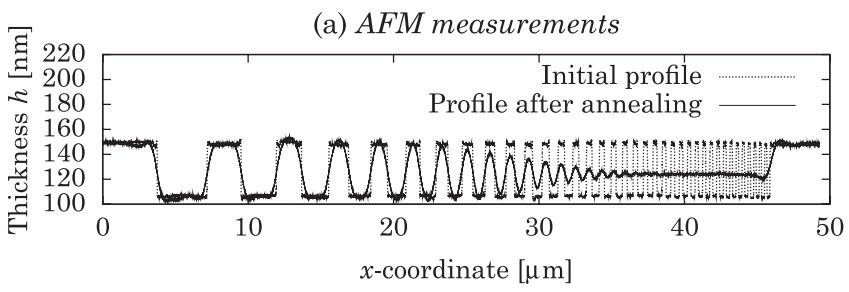

(b) Kernel function

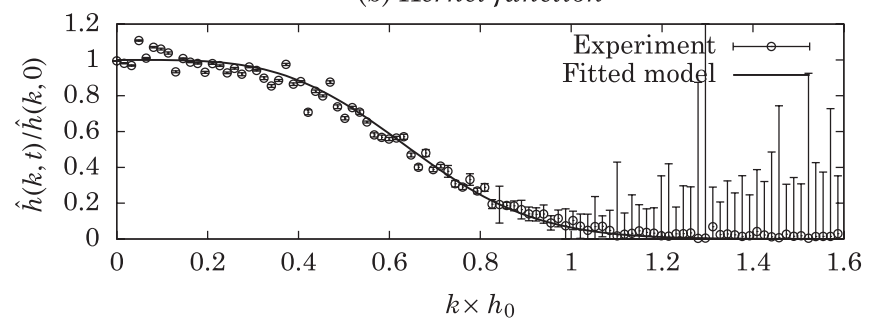

FIG. 3. Single-temperature measurement example. Polystyrene $\left(M_{w}=30 \mathrm{~kg} \cdot \mathrm{mol}^{-1}\right)$ was annealed at $120^{\circ} \mathrm{C}$ during 5 min. (a) AFM measurements of the imprinted profile before and after annealing. (b) Kernel function of the reflow, experimental points, and fitted model $\exp [-t / \tau(k)]$ as defined by Eq. (8). The fitted value is $\eta=1.0 \pm 0.1 \times 10^{6} \mathrm{~Pa} \cdot \mathrm{s}$.

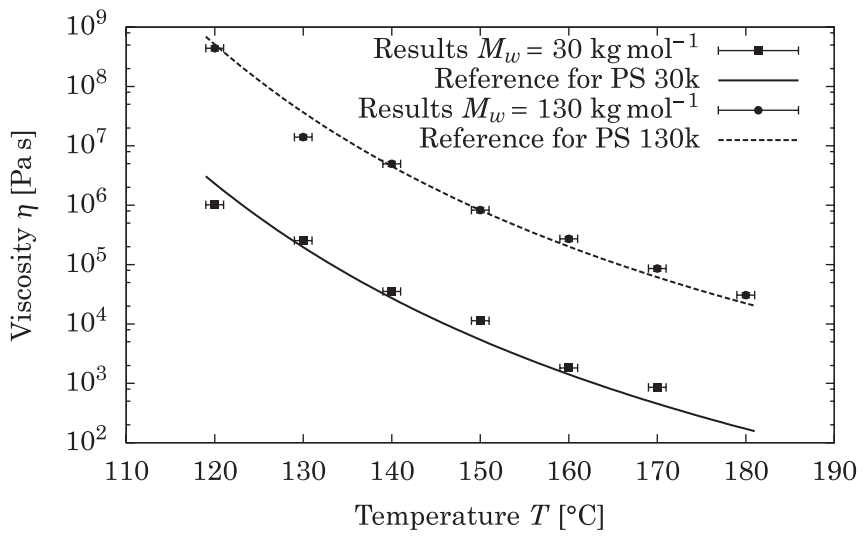

FIG. 4. Viscosity measurements for polystyrene of two molecular weights. The experimental points are plotted along with reference curves [32].

surface tension, and $\sigma_{t}=1 \%$ for the duration of the reflow. The way the uncertainty of the mean thickness influences the final result is not straightforward since it plays a role both in the fitting [through $f\left(k h_{0}\right)$ ] and in the number Ca. Still, it is possible to estimate an upper bound of this error by recalling the fact that the dynamics of the film is independent of its thickness for high-order modes $\left(k h_{0} \gg 1\right)$ and varies as $h_{0}^{3}$ for low-order modes (lubrication). Keeping the most significant dependency, we can finally write

$$
\sigma_{\eta}=\overline{\sigma_{\mathrm{Ca}}^{2}+\sigma_{\gamma}^{2}+\sigma_{t}^{2}+\left(3 \sigma_{h_{0}}\right)^{2}}=10 \% .
$$

The uncertainty of the surface tension is the main factor of the final uncertainty, and it is clear that a dedicated equipment to accurately measure the surface tension could be worthily employed. To conclude, the result of the fit for this particular example of PS $30 \mathrm{k}$ at $120^{\circ} \mathrm{C}$ is $\eta=1.0 \pm 0.1 \times 10^{6} \mathrm{~Pa} \cdot \mathrm{s}$.

Finally, the viscosity of polymer melts can be extracted using the previously described technique at various temperatures. Results for PS $30 \mathrm{k}$ and $130 \mathrm{k}$ between $120^{\circ} \mathrm{C}$ and $180{ }^{\circ} \mathrm{C}$ are reported in Fig. 4. The reference curves are obtained from the review by Kim et al. [32] on bulk polystyrene rheology. Good agreement can be seen between viscosity measurements and reference values.

The temperature dependency of viscosity corresponds to a well-known behavior of a polymer material validated at macroscopic scale (polymer bulks), extensively studied by Williams et al. [33]. These authors proposed a constitutive law describing thermal behavior for most amorphous polymers, now known as the WLF law:

$$
\frac{\eta(T)}{\eta_{s}}=\exp \left(-c_{1} \frac{T-T_{s}}{c_{2}+T-T_{s}}\right),
$$

where $T_{s}$ is a reference temperature, $\eta_{s}=\eta\left(T_{s}\right)$, and $c_{1}$ and $c_{2}$ are fitting parameters. This normalization was done for our experimental data, taking $T_{s}=150^{\circ} \mathrm{C}$, and results are summed up in Fig. 5. The WLF law was fitted to our data and parameters $c_{1}=12.6 \pm 0.1$ and $c_{2}=101 \pm 1 \mathrm{~K}$ were found. Our results are consistent with data from previous authors $[17,34]$. These results also confirm that the deviation of flow properties encountered for thickness below ten nanometers 


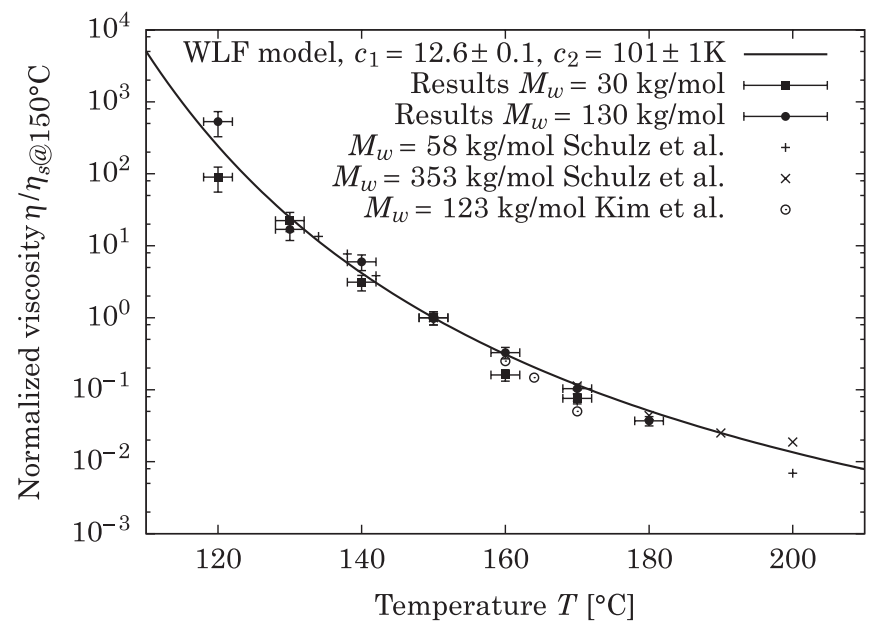

FIG. 5. Normalized polymer viscosity evolution versus temperature. Results and data from references [17,34] are normalized by the viscosity value at $150{ }^{\circ} \mathrm{C}$. WLF law with parameters $c_{1}=12.6 \pm 0.1$ and $c_{2}=101 \pm 1 \mathrm{~K}$ is also plotted (solid line).

does not occur here, since we obtained the properties of the bulk materials [6].

\section{CONCLUSION}

In this paper, we presented a method to measure the rheologic properties of polymer thin films from the reflow of nanoimprinted patterns. We derived a complete and accurate model for the evolution of the spectral density of the topology. Contrary to previous similar approaches, emphasis was put on the spatial description rather than the temporal decay of the patterns. With this model, we could develop relevant stamp designs with spatially modulated patterns to optimize experimental measurements and ensure fruitful data processing. Our approach was then successfully applied to measure the viscosity of PS $30 \mathrm{k}$ and PS $130 \mathrm{k}$ thin films. Thermal dependency of polymer viscosity was determined from reflow experiments, AFM characterizations, and spectral analysis. Consistent results were found and confirmed the presented model. Our method does not require dedicated equipment since it makes use of imprint tools and AFM. It is then a fast and cost-effective method to measure the rheologic properties of polymer thin films.

\section{APPENDIX A: CALCULATION OF THE DISPERSION RELATION}

In this section, we calculate the dispersion relation (7). We first compute $(2)^{\prime}-i \mathbf{k}(3)$ and get

$$
-k^{2} \hat{\mathbf{u}}^{\prime}+\hat{\mathbf{u}}^{\prime \prime \prime}+i k^{2} \hat{v}_{z} \mathbf{k}-i \hat{v}_{z}^{\prime \prime} \mathbf{k}=\mathbf{0} .
$$

We take the scalar product by $\mathbf{k}$ and then mass conservation yields

$$
\hat{v}_{z}^{\prime \prime \prime \prime}-2 k^{2} \hat{v}_{z}^{\prime \prime}+k^{4} \hat{v}_{z}=0 .
$$

Equation (A1) is directly solved in

$$
\hat{v}_{z}(z)=P_{1}(z) \cosh k z+P_{2}(z) \sinh k z,
$$

where $P_{1}$ and $P_{2}$ are unknown polynomials of degree one (i.e., linear functions),

$$
P_{1}(z) \equiv A_{1}+B_{1} z \quad \text { and } \quad P_{2}(z) \equiv A_{2}+B_{2} z
$$

and where we have four unknown constants $A_{1}, B_{1}, A_{2}$, and $B_{2}$. We use the property $P_{1}^{\prime \prime}=P_{2}^{\prime \prime}=0$ to write

$$
\begin{aligned}
\hat{v}_{z}^{\prime} & =\left(B_{1}+k P_{2}\right) \cosh k z+\left(B_{2}+k P_{1}\right) \sinh k z, \\
\hat{v}_{z}^{\prime \prime} & =\left(2 k B_{2}+k^{2} P_{1}\right) \cosh k z+\left(2 k B_{1}+k^{2} P_{2}\right) \sinh k z, \\
\hat{v}_{z}^{\prime \prime \prime} & =\left(3 k^{2} B_{1}+k^{3} P_{2}\right) \cosh k z+\left(3 k^{2} B_{2}+k^{3} P_{1}\right) \sinh k z .
\end{aligned}
$$

From Eq. (2) and Eq. (1) we deduce the pressure:

$$
\begin{aligned}
\frac{\omega}{i G} \hat{p} k^{2} & =-k^{2} \hat{v}_{z}^{\prime}+\hat{v}_{z}^{\prime \prime \prime}, \\
\frac{\omega}{2 i G} \hat{p} & =B_{1} \cosh k z+B_{2} \sinh k z .
\end{aligned}
$$

In order to find a relationship between the constants $B_{1}$ and $B_{2}$, we use the boundary conditions on velocity and shear stress. Condition (4) on velocity implies

$$
A_{1}=0 \quad \text { and } \quad A_{2}=-\frac{B_{1}}{k} .
$$

We use this result to rewrite $P_{1}$ and $P_{2}$ at $z=h_{0}$ :

$$
P_{1}\left(h_{0}\right)=B_{1} h_{0} \quad \text { and } \quad P_{2}\left(h_{0}\right)=-\frac{B_{1}}{k}+B_{2} h_{0} .
$$

Now we consider condition (6). We have

$$
\left(k h_{0} B_{1}+B_{2}\right) \cosh k h_{0}+k h_{0} B_{2} \sinh k h_{0}=0 .
$$

We are now able to write $B_{2}$ as a function of $B_{1}$ :

$$
B_{2}=-\frac{k h_{0} \cosh k h_{0}}{\cosh k h_{0}+k h_{0} \sinh k h_{0}} B_{1} .
$$

We now use the boundary conditions involving the free interface $\hat{h}(\mathbf{k}, \omega)$ and apply the usual properties of the hyperbolic functions to write

$$
\begin{aligned}
-i \omega \hat{h}(\mathbf{k}, \omega) & =P_{1}\left(h_{0}\right) \cosh k h_{0}+P_{2}\left(h_{0}\right) \sinh k h_{0} \\
& =B_{1} h_{0} \frac{k h_{0}-\sinh k h_{0} \cosh k h_{0}}{k h_{0}\left(\cosh k h_{0}+k h_{0} \sinh k h_{0}\right)} .
\end{aligned}
$$

In addition, from Eq. (A2) and condition (5) on the pressure, we get

$$
\begin{aligned}
\left(\gamma k^{2}+\frac{A}{2 \pi h_{0}^{4}}\right) \hat{h}(\mathbf{k}, \omega) & =2 \frac{i G}{\omega}\left(B_{1} \cosh k h_{0}+B_{2} \sinh k h_{0}\right) \\
& =2 \frac{i G}{\omega} B_{1} \frac{\cosh ^{2} k h_{0}}{\cosh k h_{0}+k h_{0} \sinh k h_{0}} .
\end{aligned}
$$

By canceling $B_{1}$, we get

$$
\begin{aligned}
& {\left[\frac{k h_{0}}{h_{0}\left(k h_{0}-\sinh k h_{0} \cosh k h_{0}\right)}-\left(\gamma k^{2}+\frac{A}{2 \pi h_{0}^{4}}\right)\right.} \\
& \times \frac{1}{2 G(\omega) \cosh ^{2} k h_{0}} \hat{h}(\mathbf{k}, \omega)=0 .
\end{aligned}
$$

The expression written between brackets is the dispersion relation we are looking for and which is reformulated in Eq. (7). 
[1] S. Y. Chou, P. R. Krauss, and P. J. Renstrom, Appl. Phys. Lett. 67, 3114 (1995).

[2] S. Merino, A. Retolaza, H. Schift, and V. Trabadelo, Microelectron. Eng. 85, 877 (2008).

[3] S. Landis, N. Chaix, C. Gourgon, C. Perret, and T. Leveder, Nanotechnology 17, 2701 (2006).

[4] S. Landis, N. Chaix, C. Gourgon, and T. Leveder, Nanotechnology 19, 125305 (2008).

[5] Y. Hirai, S. Yoshida, and N. Takagi, J. Vac. Sci. Technol. B 21, 2765 (2003).

[6] H. Bodiguel and C. Fretigny, Phys. Rev. Lett. 97, 266105 (2006).

[7] C. Redon, F. Brochard-Wyart, and F. Rondelez, Phys. Rev. Lett. 66, 715 (1991).

[8] J. Masson and P. F. Green, Phys. Rev. E 65, 031806 (2002).

[9] C. Li et al., Macromolecules 38, 5144 (2005).

[10] H. Bodiguel and C. Fretigny, Eur. Phys. J. E 19, 185 (2006).

[11] W. Ren and E. Weinan, Phys. Fluids 19, 022101 (2007).

[12] P. J. Feibelman, Langmuir 22, 2136 (2006).

[13] R. C. Major, J. E. Houston, M. J. McGrath, J. I. Siepmann, and X. Zhu, Phys. Rev. Lett. 96, 177803 (2006).

[14] A. Mechler, B. Piorek, R. Lal, and S. Banerjee, Appl. Phys. Lett. 85, 3881 (2004).

[15] M. Papi, G. Maulucci, G. Arcovito, P. Paoletti, M. Vassalli, and M. D. Spirito, Appl. Phys. Lett. 93, 124102 (2008).

[16] A. A. Svintsov, O. V. Trofimov, and S. I. Zaitsev, J. Vac. Sci. Technol. B 25, 2435 (2007).

[17] H. Kim, A. Rühm, L. B. Lurio, J. K. Basu, J. Lal, D. Lumma, S. G. J. Mochrie, and S. K. Sinha, Phys. Rev. Lett. 90, 068302 (2003).
[18] Z. Jiang, H. Kim, X. Jiao, H. Lee, Y. Lee, Y. Byun, S. Song, D. Eom, C. Li, M. H. Rafailovich, L. B. Lurio, and S. K. Sinha, Phys. Rev. Lett. 98, 227801 (2007).

[19] Z. Yang, C. Lam, E. DiMasi, N. Bouet, J. Jordan-Sweet, and O. K. C. Tsui, Appl. Phys. Lett. 94, 251906 (2009).

[20] M. Hamdorf and D. Johannsmann, J. Chem. Phys. 112, 4262 (2000).

[21] R. L. Jones, T. Hu, C. L. Soles, E. K. Lin, R. M. Reano, S. W. Pang, and D. M. Casa, Nano Lett. 6, 1723 (2006).

[22] T. Leveder, S. Landis, and L. Davoust, Appl. Phys. Lett. 92, 013107 (2008).

[23] T. Leveder, S. Landis, N. Chaix, and L. Davoust, J. Vac. Sci. Technol. B 28, 1251 (2010).

[24] T. M. Squires and S. R. Quake, Rev. Mod. Phys. 77, 977 (2005).

[25] A. Oron, S. H. Davis, and S. G. Bankoff, Rev. Mod. Phys. 69, 931 (1997).

[26] R. V. Craster and O. K. Matar, Rev. Mod. Phys. 81, 1131 (2009).

[27] M. L. Henle and A. J. Levine, Phys. Rev. E 75, 021604 (2007).

[28] J. Bicerano, Prediction of Polymer Properties (CRC Press, 2002).

[29] J. N. Israelachvili, Intermolecular and Surface Forces, 2nd ed. (Academic Press, 1991).

[30] D. Keller, Surf. Sci. 253, 353 (1991).

[31] C. Hahlweg, M. Gruhlke, and H. Rothe, Meas. Sci. Technol. 20, 084018 (2009).

[32] S. H. Kim, F. Teymour, and J. A. Debling, J. Appl. Polym. Sci. 103, 2597 (2007).

[33] M. L. Williams, R. F. Landel, and J. D. Ferry, J. Am. Chem. Soc. 77, 3701 (1955).

[34] H. Schulz, M. Wissen, N. Bogdanski, H. Scheer, K. Mattes, and C. Friedrich, Microelectron. Eng. 78-79, 625 (2005). 